\title{
Use of microperimetry to evaluate Hydroxychloroquine and Chloroquine retinal toxicity
}

Lucía Martínez-Costa M.D., Ph.D. ${ }^{\text {a,* }}$, María Victoria Ibañez Ph.D. ${ }^{\text {, }}$, Cinta Murcia-Bello M.D. ${ }^{a}$, Irene Epifanio Ph.D. ${ }^{b}$, Cristina Verdejo-Gimeno M.D., Ph.D. ${ }^{\text {, }}$ Emma Beltrán-Catalán M.D. ${ }^{c}$, Pilar Marco-Ventura M.D., Ph.D. ${ }^{\text {a }}$

${ }^{a}$ Department of Ophthalmology. Hospital Dr. Peset. Avda. Gaspar Aguilar, 90. E-46017. Valencia. Spain. Tel.: 00341622566. FAX: 00341622501

${ }^{b}$ Department of Mathematics. Universitat Jaume I.E-12071- Castellón. Spain

${ }^{c}$ Department of Rheumatology. Consorcio Hospital General. Av de las Tres Cruces 0, E-46014 Valencia. Spain

\footnotetext{
*Corresponding author

Email addresses: martinezcosta_luc@gva.es (Lucía Martínez-Costa M.D., Ph.D. ), mibanez@mat.uji.es (María Victoria Ibañez Ph.D.), c.murciabello@gmail.com (Cinta Murcia-Bello M.D.), epifanio@mat.uji.es (Irene Epifanio Ph.D.), verdejo_cri@gva.es (Cristina Verdejo-Gimeno M.D., Ph.D.), beltran_emm@gva.es (Emma Beltrán-Catalán M.D.), marco_pilven@gva.es (Pilar Marco-Ventura M.D., Ph.D.)
} 


\section{Abstract}

Objective: To check the ability of microperimetry to detect early retinal damage in rheumatic patients taking hydroxychloroquine (HCQ) and/or chloroquine (CQ). To describe the microperimetric alterations attributable to these drugs and their correlation with some clinical variables.

Design: Controlled cross-sectional study.

Participants: Patient group: 209 patients taking HCQ or CQ. Control group: 204 individuals not under antimalarials. Exclusion criteria: other diseases that could alter microperimetry.

Methods: An ophthalmic examination and a microperimetry were performed on all individuals. Outcomes: Average threshold (AT), Fixation stability (FS) and Macular integrity (MI). For the patients, information about their weight, height, main diagnosis, daily and cumulative dose, creatinine, bilirubin and transaminases levels were collected. ANOVA, t-tests and a regression analysis were carried out to detect differences between groups.

Results: Significant differences in microperimetry indexes were detected between cases and controls, between patients of different age groups, and between patients taking CQ and HCQ. Significant differences were also detected in retinal sensitivity between patients overdosed for CQ but not for those overdosed for HCQ. Daily overdosing per ideal weight alone cannot explain retinal toxicity, although the effect of cumulative dose in macular sensibility is significant to explain both AT and MI.

Conclusions: Microperimetry is an accurate tool for detecting early macular hyposensibility caused by CQ and HCQ. Microperimetry indexes of retinal sensibility are worse in elderly patients taking these drugs, and in 
short stature patients taking CQ. A high cumulative dose is an important factor in explaining retinal hyposensibility on microperimetry. 


\section{Introduction}

Chloroquine (CQ) and its analogue, hydroxychloroquine (HCQ) are antimalarial drugs that have been used as treatment of various rheumatological and dermatological diseases including systemic lupus erythematosus (SLE), rheumatoid arthritis (RA) and chronic discoid lupus. ${ }^{1}$ However, retinal toxicity has been described. Although the risk of toxicity from CQ and HCQ is low, many thousands of individuals are taking these drugs. After 5 to 7 years of use the prevalence increases to $1 \% .^{2}$ The risk of toxicity depends on cumulative exposure (a cumulative dose of $1000 \mathrm{~g}$ of HCQ, which is reached in 7 years with a typical daily dose of $400 \mathrm{mg}$, and a cumulative dose of $460 \mathrm{~g}$ of $\mathrm{CQ}$, which is reached in 5 years with a typical daily dose of $250 \mathrm{mg}$ ). Other risk factors are renal or liver diseases, underlying retinal disease and age. The clinical sign of CQ and HCQ toxicity is characterized by a ring of retinal pigment epithelium depigmentation, often sparing the foveal center, known as a bull's eye maculopathy. Paracentral scotomas appear before changes are seen on a fundus examination. Drug use cessation at this stage of early functional loss might prevent future visual loss, but after maculopathy develops, cessation of the drug does not show clinical recovery. ${ }^{3}$ Recommendations on screening for CQ and HCQ retinopathy are automated visual field and, where available, testing with one or more of the recommended objective tests: spectral domain-OCT (SD-OCT), multifocal electroretinogram (mfERG) or fundus autofluorescence (FAF). ${ }^{4,5}$ However, visual field accuracy relies on patient's cooperation, and the objective procedures are not readily available in many offices. The goal of screening is to recognize toxicity before a severe degree of visual field loss occurs. Nowadays, there is no established a gold 
standard test for screening.

Microperimetry consists in testing perimetry under simultaneous visualization of the fundus. Exact correlation between retinal pathology and functional alteration is obtained. It allows a precise evaluation of macular sensitivity, providing an accurate detection of small scotomic areas in terms of their position, extension and severity in the macular area with a real-time correction of eye movements. ${ }^{6}$ Its uses are both clinical and for research. ${ }^{7,8,9,10,11}$ In the present study we assess CQ and HCQ toxicity using microperimetry. Our purpose is to describe the value of microperimetry as a high sensitivity test for the screening of CQ and HCQ retinopathy. We study wheather macular sensibility indexes provided by microperimeter are decreased in our patients with respect to persons not taking antimalarials. Moreover, we look for the correlation between microperimetry abnormalities and the main clinical variables influencing retinal damage.

\section{Materials and methods}

All patients and controls gave written informed consent to participating in the study, which was approved by the ethical committee of our hospital (code 09/046). Moreover, the procedures complied with the tenets of the Declaration of Helsinki.

A total of 413 individuals have been included in this cross-sectional casecontrol study. We studied 209 patients taking CQ and HCQ. In addition, 204 individuals not being treated with antimalarials were included as controls. Controls were chosen from healthy volunteers (57\%) and from patients affected by rheumatic diseases that have never been managed with antimalar- 
ials (43\%). In the patient group we obtained data about weight, height, diagnosis, daily doses, months under treatment and cumulative dose. A complete ophthalmological examination was performed in patients and controls which included visual acuity, biomicroscopy, intraocular pressure and funduscopy. The presence of corneal drug deposits attributable to CQ and HCQ was recorded. Data from three consecutive blood analyses (creatinine, bilirubin and transaminases AST/ALT) were also obtained in order to identify renal or liver disfunction that could increase $\mathrm{CQ}$ and HCQ retinal toxicity. Patients suffering from other diseases that could alter the fundus perimetry such as glaucoma, gross ametropia, macular drusen, other maculopathies etc were excluded. Finally, a microperimetry was done using the expert exam strategy of MAIA microperimeter (CenterVue SpA, Padova Italy). Then we recorded three indexes provided by the microperimeter: Average threshold (AT), Fixation stability (FS) and Macular integrity (MI). A stimulus intensity ranged from 0 to $36 \mathrm{~dB}$. A predefined grid of 37 points and 30 macular coverage was used. Threshold sensitivities at each predefined point were calculated using a staircase 4-2 strategy. A patient's AT results were compared with age-adjusted normative data. Two variables in the data set measured patients' fixation stability: FS p1 and FS p2. Both variables measure the percentage of fixation points located within a circle centered on the gravitational center of all fixation points. The difference between them arises on the diameter of their respective circles, the diameter for FSp2 being greater than for FSp1. MI is an index of macular health that is calculated using a neural network multivariate model (see section 4 for a detailed explanation). Best-corrected visual acuity (BCVA) was measured in decimal units 
on a decimal chart. The results were then converted to the logarithm of the minimum angle of resolution scale (log MAR).

\section{Results}

All the exams with less than $75 \%$ reliability were excluded, finally resulting in 200 controls and 194 cases. Table 1 shows the demographics of our sample. Regarding the patient group, Table 2 shows the main variables collected in a more detailed way. A total of 17 (8.76\%) cases were diagnosed as having a toxic maculopathy (see Table 3) considering clinical and microperimetrical findings: 7 of them were considered as probable maculopathy and 10 $(5.15 \%)$ as definite toxic maculopathy (see Figure 1). Probable maculopathy was diagnosed if the patient had a pericentral scotoma with three or more adjacent points between two and three standard deviations from the normal average (in yellow in Figure 1) and/or two or more adjacent points with a sensitivity beyond three standard deviations from the normal average (in orange, red and black in Figure 1). Definite maculopathy was diagnosed if the patient also had characteristic pigmentary changes of antimalarial toxicity. Among these 10 patients, 4 had complete bull's eye maculopathy and 6 had only subtle sectorial depigmentation of the macular area. FS and BCVA were good (less than log MAR 0.2) in 9 patients having definite maculopathy. The remaining patient had an advanced bull-eye maculopathy with foveal involvement: unstable fixation and BCVA 0.5 log MAR. All 10 patients diagnosed as having definite maculopathy were women, and all were less than $163 \mathrm{~cm}$ in height. In addition, 3 patients presented corneal drug deposits. All three were under CQ therapy: one was diagnosed with definite CQ maculopathy 
(cumulative dose $=548 \mathrm{~g}$ ) and the other was diagnosed as probable CQ maculopathy (cumulative dose $=274 \mathrm{~g}$ ). In these two patients, corneal deposits disappeared a few weeks after stopping antimalarials.

As there were no significant differences between AT and MI indexes corresponding to the right and left eye of each patient, from now on, when talking about AT and MI values, we will refer to the mean value between both eyes for each patient.

Significant differences in AT between cases and controls were detected (see Table 4). However, MI was higher in controls. This result seemed paradoxical, because higher MI suggests a greater likelihood of abnormal findings. In trying to investigate if a higher MI value in controls could correspond to a lower fixation stability, this variable was also analyzed (Table 4). Nevertheless, no significant differences were detected between the mean fixation stability (FSp1 and FSp2) of controls and patients. If age stratification was done, we could see that MI was significatively higher in individuals under 60 years-old than in patients, but over 61, patients taking antimalarials had higher MI than healthy controls (Table 5).

We did not find significant differences in AT or in MI with respect to liver and renal functional blood analysis. The creatinine, transaminases and bilirrubine levels of our patients did not show any correlation with macular hyposensibility on microperimetry.

An ANOVA and a Tukey test were done in order to establish if there were significant differences in microperimetry indexes between patients taking CQ, HCQ or both drugs. As shown in Table 6, significant differences in retinal sensitivity (AT and MI) were detected between patients taking CQ 
and HCQ, but there were no differences in the patients that had been managed successively with both drugs with respect to those managed just with one drug.

As CQ and HCQ are not retained in fatty tissues, we calculated the daily dose per $\mathrm{kg}$ of ideal weight and looked for its relationship with macular sensibility indexes. Our conclusion was that a daily overdose per ideal weight alone cannot explain retinal toxicity (Fig. 2). However, using the cut-off value of toxic doses traditionally considered in medical literature, i.e more than $3 \mathrm{mg} / \mathrm{kg} /$ day for CQ and more than $6.5 \mathrm{mg} / \mathrm{kg} /$ day for HCQ, we found significant differences in retinal sensitivity between patients overdosed for CQ but not for those overdosed for HCQ. We also checked if macular toxicity was influenced by our patients' ages. Three age groups were considered: under 40, between 41 and 60 and over 60 years-old. In our sample, patients over 60 under treatment with CQ and HCQ presented worse sensitivities in microperimetry indexes. In fact, when the effect of daily dose per ideal weight, months under treatment and age were considered together, the most important variable that influences macular damage was the patient's age (adjusted R-squared linear regression=0.6628). Finally, we looked for the effect of the cumulative dose of antimalarials on macular sensibility. Again, we found that patients' indexes AT and MI were worse for higher values of cumulative dose, and that this effect was greater for CQ than for HCQ. When considering a model including cumulative doses, age, height and age as explicative covariates, we found that age, height and cumulative dose of $\mathrm{CQ}$ affected AT independently (adjusted R-squared linear regression=0.3187). Age and cumulative dose of CQ were also related to MI (adjusted R-squared 
linear regression $=0.6353)$.

\section{Discussion}

The incidence of toxic maculopathy due to CQ and HCQ varies greatly. More accurate diagnostic methods, or the combination of objective and subjective tests, will allow an earlier diagnosis and probably an increase in the incidence ratios. It must be taken into account that bull's eye maculopathy is an advanced sign, and that incidence ratios should also include early cases of retinal toxicity. These cases are sometimes difficult to diagnose because pericentral scotomata can be produced by a lot of clinical entities that can damage the macula in addition to or instead of antimalarials. As retinal damage will not reverse after stopping the drug, the clinician cannot always be sure if they were the cause of maculopathy or not.

The appearance of corneal drug deposits in patients under CQ and HCQ is well known. ${ }^{1}$ Confocal microscopy has recently been used to detect these deposits that correlate with high cumulative doses. ${ }^{12}$ In this study, we found slit lamp visible deposits in three patients, all three under CQ. Two of them also presented CQ maculopathy.

In this paper we look for differences in global indexes provided by microperimetry between a group of patients taking CQ, HCQ or both, and a group of untreated persons. We found that the average threshold of retinal sensitivities at the predetermined points near the foveal center were significatively lower in cases than in controls. This would indicate that antimalarials induce a global hyposensibility at the macula that does not appear in a similar group of population who never took those tablets. All except one of our 
patients had good BCVA and stable fixation on microperimetry, and the vast majority had no pigmentary changes. This corroborates the fact that a loss of central vision and anatomical changes are advanced signs that should not be used for screening purposes. ${ }^{4}$ The other index studied (MI) shows a paradoxical finding, because for individuals under 60 it is significatively higher, i.e more impaired, in controls than in patients. In order to look for some explanation of this result, let us analyze the composition of these two groups. In all patients one or more conventional macular perimetry were performed previously because they were included in our clinic's screening program of antimalarial toxicity. The control group included people without any eye disease, so they never underwent visual field testing. It is possible that the learning effect explains in part why controls have worse MI than patients. Test-retest reliability of macular fundus perimetry was checked by Chen et al. ${ }^{13} \mathrm{MI}$ uses a neural network multivariate model that includes age, average threshold value, a measure of points with threshold below $25 \mathrm{~dB}$ and all measured threshold values. MI is a numerical value that describes the likelihood that a patient's responses are normal when compared to age-adjusted normative data. The neural network has been trained on normal and pathological examinations (age-related macular degeneration). With respect to normative data used in the elaboration of the MAIA neural network (Smolek et al, Neural Network Algorithms for a Device to Measure Macular Visual Sensitivity. Scientific poster. ARVO Meeting 2010), our patients and controls had lower AT. A neural network is a classifier where the aforementioned inputs are used to obtain that likelihood. Unlike other classifiers such as the classical linear discriminant, neural networks are black boxes, and you cannot know which 
variables are the most discriminative. Furthermore, care should be taken with the training to avoid overlearning, i. e. a good performance with the training sample but with a poor generalization with unseen samples. The images of our young controls with high MI were revised and we found that they had a threshold below $25 \mathrm{~dB}$ in a few points, which probably produced those high MI despite having good average threshold. Furthermore, in those cases, the MI difference between both eyes was high, which was not so frequent among the patients. Larger prospective studies must be done in order to validate $\mathrm{MI}$ as a good index for evaluating other diseases distinct from age-related maculopathy.

In our sample, mean age and cumulative dose are significatively higher for patients under CQ and both drugs than in patients that are treated with HCQ only. In Spain, HQC was not available until 2002. Moreover, the first commercially available presentation of HCQ presented some problems of tolerance, ${ }^{14}$ and some patients were either switched back to CQ or their physicians postponed the use of HCQ until this problem was solved some years later. This is why in our sample patients under HCQ are younger and their cumulative doses are smaller than those treated with CQ. This bias can explain why we found a low incidence of maculopathy in patients taking just $\mathrm{HCQ}$, even though a greater retinal toxicity for CQ has largely been evidenced. ${ }^{15}$ However, it has been considered that the increased toxicity rates of CQ could be due to pill size for chloroquine (250 mg), which makes overdosing much easier than with HCQ. ${ }^{16}$ In fact, all patients measuring less than $160 \mathrm{~cm}$ would be at risk of CQ toxicity because of overdosing. ${ }^{2}$ In our study, $80 \%$ of patients diagnosed as having toxic maculopathy were overdosed 
for CQ, and all of them were of short stature.

As anatomical changes seen on funduscopy appear late, new imaging techniques have been used to improve early diagnosis, such as fundus autofluorescence and sprectral-domain OCT. Electrophysiological tests of retinal function, mainly mfERG, is also considered in early diagnosis or as an additional tool to confirm doubtful cases. ${ }^{17}$ However, in a recent paper, ${ }^{18} \mathrm{mfERG}$ failed to detect as much as approximately $28 \%$ of individuals with hydroxychloroquine retinal toxicity. False negatives appear to be even higher for SD-OCT than for mfERG testing. Evidence about the usefulness of FAF in early diagnosis is lacking. ${ }^{18,19}$ Macular perimetry, funduscopic examination and photography continue to provide the highest yields of early cases. ${ }^{17}$ It has been recently considered that revised guidelines, ${ }^{4}$ emphasizing mfERG, SD-OCT or FAF, raised screening cost without improving case detection. ${ }^{16}$ We believe that fundus microperimetry provides more detailed information about retinal sensibility at the macular area and gives the clinician a precise correlation with anatomical changes. Our results indicate that microperimetry is a very good test to detect early and subtle functional impairment caused by CQ and HCQ.

CQ and HCQ are effective drugs in the management of severe rheumatic disease, but in order to avoid retinal damage, dosage must be careful, particularly in short patients who are expected to be treated for many years. Advanced age is an important risk factor for toxicity. ${ }^{17}$ To the best of our knowledge, this is the first study that uses microperimetry as a screening test for retinal toxicity because of antimalarials. In fact, this is the first work that uses microperimetry in a sample of more than 200 individuals. 


\section{Conclusions}

Microperimetry is an accurate tool for detecting early macular damage associated with CQ and HCQ therapy. Patients taking CQ, HCQ or both show reduced threshold retinal sensibility in the macular area. High HCQ and/or CQ cumulative doses, advanced age, daily CQ overdosing per ideal weight, and short stature are independently associated with worse macular indexes on microperimetry. There are several limitations to the present study. Larger prospective studies must be carried out in order to confirm the presence of macular hyposensibility over time. Future directions will be the description of spatial localization and characterization of early central scotomas induced by CQ and HCQ.

\section{Acknowledgements}

The authors declare that they have no financial and (or) proprietary interests.

This work has been partially funded by projects: PI09/90687 of the Instituto de Salud Carlos III, Spanish Ministry of Science and Innovation (FEDER funds), GV/2011/004 of the Generalitat Valenciana and P11A2011-11 of UJIBancaixa .

\section{References}

[1] Tehrani R, Ostrowski R, Hariman R, Jay W. Ocular toxicity of hydroxychloroquine. Semin Ophthalmol. 2008;23(3):201-9. 
[2] Wolfe F, Marmor M. Rates and predictors of hydroxychloroquine retinal toxicity in patients with rheumatoid arthritis and systemic lupus erythematosus. Arthritis Care Res (Hoboken). 2010;62(6):775-84.

[3] Michaelides M, Stover N, Francis P, Weleber R. Retinal Toxicity Associated With Hydroxychloroquine and Chloroquine. Risk Factors, Screening, and Progression Despite Cessation of Therapy. Arch Ophthalmol. 2011;129(1):30-39.

[4] Marmor M, Kellner U, Lai T, Lyons J, Mieler W. Revised recommendations on screening for chloroquine and hydroxychloroquine retinopathy. Ophthalmology. 2011;118(2):415-22.

[5] Xiaoyun M, Dongyi H, Linping H. Assessing chloroquine toxicity in RA patients using retinal nerve fibre layer thickness, multifocal electroretinography and visual field test. Br J Ophthalmol. 2010 Dec;94(12):1632-6.

[6] Rohrschneider K, Bltmann S, Springer C. Use of fundus perimetry (microperimetry) to quantify macular sensitivity. Prog Retin Eye Res. 2008 Sep;27(5):536-48.

[7] Angi M, Romano V, Valldeperas X, Romano F, Romano M. Macular sensitivity changes for detection of chloroquine toxicity in asymptomatic patient. Int Ophthalmol. 2010 Apr;30(2):195-7.

[8] Fujita K, Shinoda K, Matsumoto C, Imamura Y, Tanaka E, Mizutani $\mathrm{Y}$, et al. Microperimetric evaluation of chronic central serous chori- 
oretinopathy after half-dose photodynamic therapy. Clin Ophthalmol. 2012;6:1681-7.

[9] Nittala M, Gella L, Raman R, Sharma T. Measuring retinal sensitivity with the microperimeter in patients with diabetes. Retina. 2012 Jul;32(7):1302-9.

[10] Sulzbacher F, Kiss C, Kaider A, Eisenkoelbl S, Munk M, Roberts P, et al. Correlation of SD-OCT features and retinal sensitivity in neovascular age-related macular degeneration. Invest Ophthalmol Vis Sci. 2012 Sep;53(10):6448-55.

[11] Lee K, Markowitz S. Scotoma size reduction as an adaptive strategy in age-related macular degeneration. Can J Ophthalmol. 2010 Aug;45(4):393-8.

[12] Ma X, He L, He D, Xu J. Chloroquine keratopathy of rheumatoid arthritis patients detected by in vivo confocal microscopy. Curr Eye Res. 2012 Apr;37(4):293-9.

[13] Chen F, Patel P, Xing W, Bunce C, Egan C, Tufail A, et al. Testretest variability of microperimetry using the Nidek MP1 in patients with macular disease. Invest Ophthalmol Vis Sci. 2009 Mar;50(7):346472.

[14] Jiménez-Alonso J, Sabio J, Carrillo-Alascio P, Jiménez-Jimez J, OrtegoCenteno N, Jiménez-Jáimez E, et al. Intolerance to hydroxychloroquine marketed in Spain (Dolquine) in patients with autoimmune conditions. Rev Clin Esp. 2004 Nov;204(11):588-91. 
[15] Levy G, Munz S, Paschal J, Cohen H, Pince K, Peterson T. Incidence of hydroxychloroquine retinopathy in 1,207 patients in a large multicenter outpatient practice. Arthritis and Rheumatism. 1997 Aug;40(8):14826.

[16] Browning D. Hydroxychloroquine and chloroquine retinopathy: screening for drug toxicity. Am J Ophthalmol. 2002 May;133(5):649-56.

[17] Marmor M. Comparison of screening procedures in hydroxychloroquine toxicity. Arch Ophthalmol. 2012 Apr;130(4):461-9.

[18] Farrell D. Retinal toxicity to antimalarial drugs: chloroquine and hydroxychloroquine: a neurophysiologic study. Clin Ophthalmol. 2012;6(4):377-83.

[19] Chen E, Brown D, Benz M, Fish R, Wong T, Kim R, et al. Spectral domain optical coherence tomography as an effective screening test for hydroxychloroquine retinopathy (the "flying saucer" sign). Clin Ophthalmol. 2010 Oct;21(4):1151-8.

Keywords: chloroquine; hydroxychloroquine; visual field tests; microperimetry; retinal diseases. 
Table 1: Description of the database.

\begin{tabular}{c|cc|c} 
& Controls & Cases & p-value \\
\hline Sex:(\% Female) & $84.00 \%$ & $80.42 \%$ & 0.42 \\
Age: average \pm sd & $53.74 \pm 13.37$ & $51.24 \pm 15.13$ & 0.08 \\
Weight (in Kg): average \pm sd & - & $70.15 \pm 15.35$ & - \\
Height (in cm): average $\pm \mathrm{sd}$ & - & $159.71 \pm 12.98$ & -
\end{tabular}


Table 2: Description of the group of patients. Diagnosis acronyms: systemic lupus erythematosus (SLE), rheumatoid arthritis (RA), other: undifferentiated arthritis, cutaneous diseases. Overdosed: $\mathrm{HCQ}=$ patients receiving more than $6,5 \mathrm{mg} / \mathrm{Kg}$ ideal weight/day; $\mathrm{CQ}=$ patients receiving more than $3 \mathrm{mg} / \mathrm{Kg}$ ideal weight/day. $\mathrm{CQ} \rightarrow \mathrm{HCQ}=$ Patients first on $\mathrm{CQ}$ and switched to $\mathrm{HCQ}$ over the evolution of the disease. According to the characteristics of the different variables, this table shows either the mean \pm sd values or the respective counts and percentages.

\begin{tabular}{c|ccc|c} 
& HCQ & CQ & CQ $\rightarrow$ HCQ & p-value \\
\hline$n(\%)$ & $130(67.01 \%)$ & $30(15.46 \%)$ & $34(17.52 \%)$ & \\
BCVA (LogMAR, mean) & $0.06 \pm 0.09$ & $0.10 \pm 0.13$ & $0.12 \pm 0.21$ & 0.03 \\
Diagnosis: & & & & \\
SLE & $64(68.81 \%)$ & $10(10.75 \%)$ & $19(20.43 \%)$ & \\
RA & $33(55.00 \%)$ & $15(25.00 \%)$ & $12(20.00 \%)$ & \\
Other & $32(84.21 \%)$ & $4(10.53 \%)$ & $2(5.26 \%)$ & \\
Duration of theraphy (months) & $38.29 \pm 38.88$ & $103.66 \pm 63.88$ & $121.55 \pm 59.31$ & $<0.001$ \\
Cum. HCQ dosis & $357.61 \pm 381.04$ & 0 & $503.23 \pm 445.46$ & 0.09 \\
Cum. CQ dosis & 0 & $733.53 \pm 432.96$ & $475.76 \pm 411.97$ & 0.02 \\
Overdosed (n (\%)) & $22(16.92 \%)$ & $25(83.33 \%)$ & $7(20.58 \%)$ & $<0.001$ \\
AST & $21.72 \pm 8.26$ & $22.24 \pm 7.67$ & $22.94 \pm 8.73$ & 0.73 \\
ALT & $21.37 \pm 14.58$ & $20.41 \pm 7.19$ & $22.68 \pm 17.08$ & 0.81 \\
Bilirrubin & $0.49 \pm 0.34$ & $0.56 \pm 0.32$ & $0.43 \pm 0.19$ & 0.31 \\
Creatinine & $0.79 \pm 0.23$ & $0.81 \pm 0.16$ & $0.89 \pm 0.46$ & 0.17 \\
Corneal Drug Deposits (n) & 0 & 3 & 0 &
\end{tabular}


Table 3: Characteristics of patients with toxic maculopathy.

\begin{tabular}{c|cccc} 
& HCQ & CQ & CQ $\rightarrow$ HCQ & Global \\
\hline$n(\%)$ & 2 & 10 & 5 & 17 \\
Probable maculopathy & 1 & 4 & 2 & 7 \\
Definite maculopathy & 1 & 6 & 3 & 10 \\
Age & $41.00 \pm 9.89$ & $57.00 \pm 11.56$ & $60.60 \pm 20.21$ & $56.17 \pm 14.78$ \\
Cum HCQ dosis & $200.5 \pm 180.31$ & 0 & $251.8 \pm 308.24$ & $207.50 \pm 257.88$ \\
Cum CQ dosis & 0 & $648.10 \pm 252.13$ & $689.40 \pm 763.23$ & $661.87 \pm 455.75$ \\
Overdosed (n (\%)) & $1(50.00 \%)$ & $8(80.00 \%)$ & $2(40.00 \%)$ & $11(64.70 \%)$ \\
Deposits (n (\%)) & 0 & $2(20 \%)$ & 0 & $2(11.76 \%)$
\end{tabular}




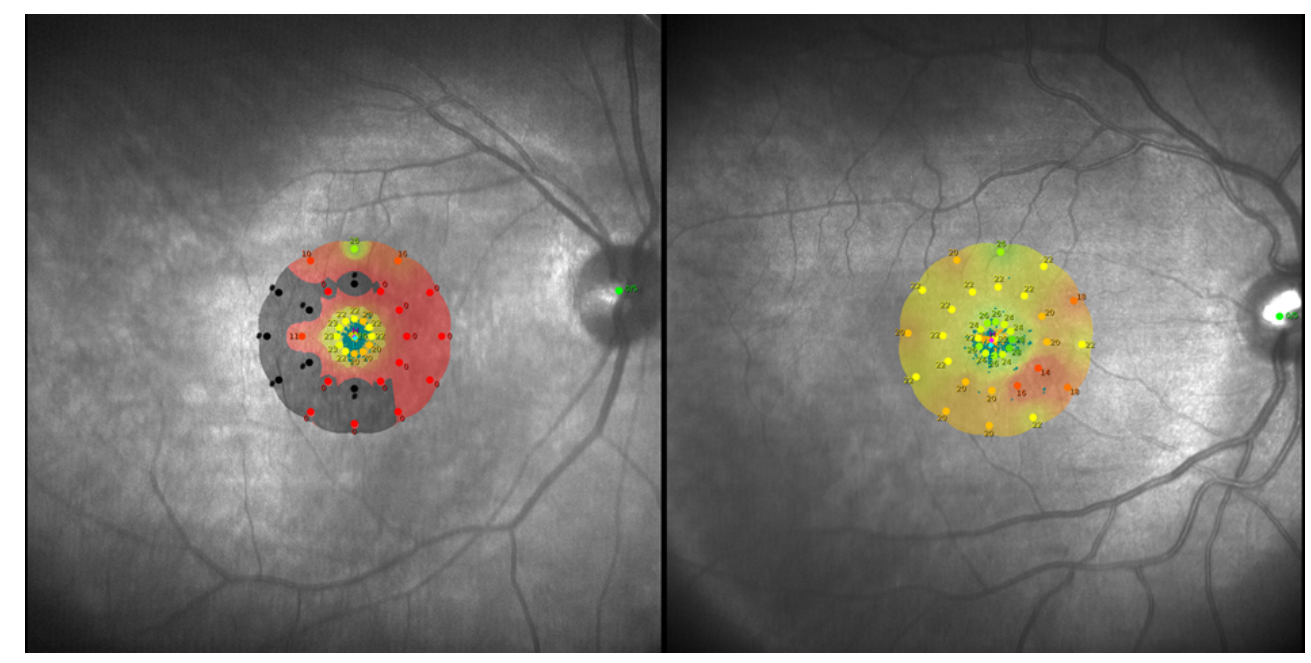

Figure 1: Interpolation maps of microperimetries corresponding to: (left) a patient diagnosed as definite maculopathy with stable fixation and good central responses but severe pericentral hyposensitivity with an absolute scotoma; (right) a patient diagnosed as probable maculopathy with stable fixation and good central sensitivity but nasal pericentral scotoma. 
Table 4: Differences between controls and cases in FS, AT and MI (sd denotes standard deviation).

\begin{tabular}{c|ccc|c} 
& Global & Cases & Controls & $\begin{array}{c}\text { p-value } \\
\text { Cases vs Controls }\end{array}$ \\
& & & & \\
\hline Mean AT & $26.83 \pm 2.27$ & $26.52 \pm 2.64$ & $27.12 \pm 1.81$ & 0.0092 \\
Mean MI & $43.56 \pm 34.02$ & $32.72 \pm 34.33$ & $54.07 \pm 30.29$ & 0 \\
Mean FS p1 & $90.83 \pm 11.57$ & $89.9 \pm 12.58$ & $91.83 \pm 10.42$ & 0.0827 \\
Mean FS p2 & $97.18 \pm 5.82$ & $96.93 \pm 5.98$ & $97.42 \pm 5.67$ & 0.4062
\end{tabular}


Table 5: Comparison of MI and FSp1 mean values between cases and controls for different ages.

\begin{tabular}{c|c|ccc|c}
\hline & & \multicolumn{3}{|c}{ Age } & \\
& & $\leq 40$ & $(40-60]$ & $>60$ & p-value \\
\hline \multirow{2}{*}{ MI } & Cases & $5.45 \pm 9.57$ & $21.75 \pm 23.26$ & $73.61 \pm 23.61$ & $<0.0001$ \\
& Controls & $26.38 \pm 26.31$ & $50.8 \pm 26.12$ & $74.77 \pm 23.11$ & $<0.0001$ \\
\hline \multirow{2}{*}{ FS p1 } & p-value & $<0.0001$ & $<0.0001$ & 0.7842 & \\
& Cases & $93.68 \pm 6.74$ & $90.87 \pm 9.68$ & $84.66 \pm 17.83$ & 0.0003 \\
& Controls & $92.66 \pm 12.01$ & $92.45 \pm 8.53$ & $90.42 \pm 11.92$ & 0.4148 \\
\hline & p-value & 0.6406 & 0.2521 & 0.0402 & \\
\hline
\end{tabular}


Table 6: Differences between AT and MI mean values depending on the drug.

\begin{tabular}{c|ccc|c} 
& HCQ & CQ & CQ $\rightarrow$ HCQ & p-value \\
\hline Mean AT & $26.90 \pm 2.08$ & $24.96 \pm 3.61$ & $26.44 \pm 3.06$ & 0.0011 \\
Mean MI & $27.78 \pm 31.26$ & $49.97 \pm 38.18$ & $36.36 \pm 37.56$ & 0.0042
\end{tabular}




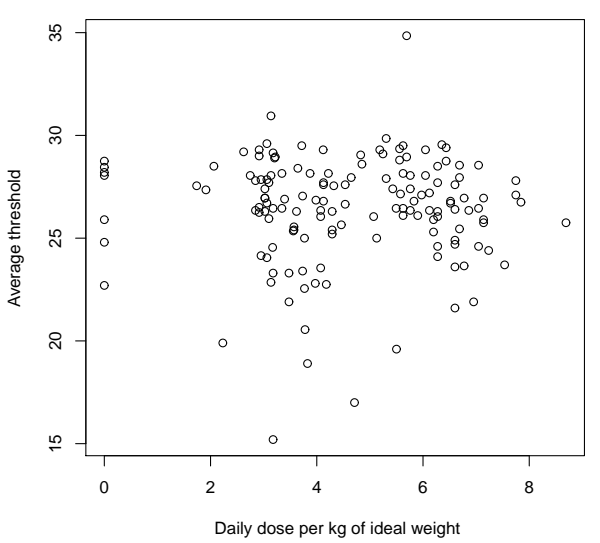

(a)

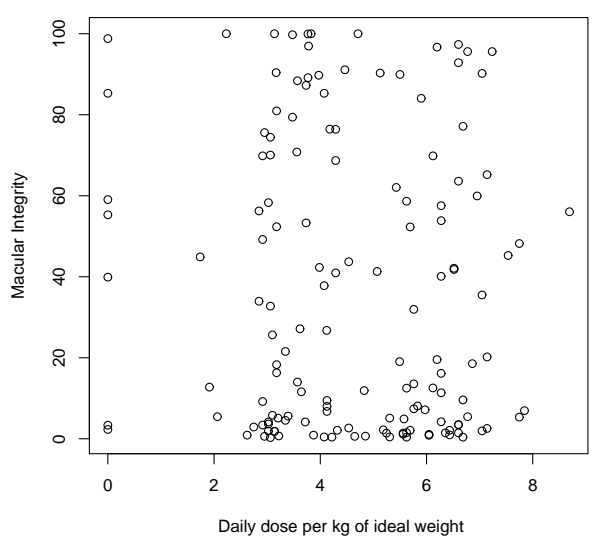

(b)

Figure 2: Daily dose per Kg of ideal weight versus (a) average threshold and (b) macular integrity. 